\title{
How to introduce fiber optics to beginners
}

\section{Bernt Sundstrom}

Bernt O. Sundstrom, "How to introduce fiber optics to beginners," Proc. SPIE 3190, Fifth International Topical Meeting on Education and Training in Optics, (8 December 1997); doi: 10.1117/12.294382

Event: Fifth International Topical Meeting on Education and Training in Optics, 1997, Delft, Netherlands 
How to introduce fiber optics to beginners

Bernt O. Sundström

Ericsson Components AB, Fiber Optics Research Center

Isafjordsgatan 16, S-164 81 KISTA-STOCKHOLM, Sweden

\begin{abstract}
Demonstrations of fiber optic transmission are described, using light emitting diodes, plastic fiber and a photodiode. Total reflection is visualized by a laser pointer and a Plexiglas bar. Rayleigh scattering can be seen from a plastic fiber. Bend loss and coupling loss can also be shown.
\end{abstract}

Keywords: fiber optics education, fiber optics demonstration

\title{
1. INTRODUCTION
}

Fiber optics is a relatively new communication technique and telecommunication engineers and technicians need education in optics and fiber properties. Optics has the advantage that the phenomena are easy to visualize, e.g. fiber optic transmission, total reflection, Rayleigh scattering, bend loss and splice loss.

\section{FIBER OPTIC TRANSMISSION}

The simplest way to demonstrate fiber optic transmission is to put one end of a plastic fiber, e.g. $1 \mathrm{~mm}$ in diameter, near a lamp and observe the light from the other end. Moving a finger in front of the first fiber end or moving the fiber itself will modulate the light, simulating signal transmission.

The principles of modulating a laser or LED by an electric signal, detect and amplify it, can be demonstrated by simple and cheap equipment, using an amplitude modulated LED, a photodiode and a $1 \mathrm{~W}$ audio amplifier. A tape recorder (cassette player) or a cheap music circuit serves as signal source for the transmitter and a loudspeaker is connected to the amplifier output. A plastic fiber is preferred to glass fiber as its large diameter and numerical aperture simplify launching and observation of light. An infrared LED demonstrates the possibilities of invisible radiation, which is used for glass fibers due to lower losses. The circuit diagram is shown in Fig. 1.

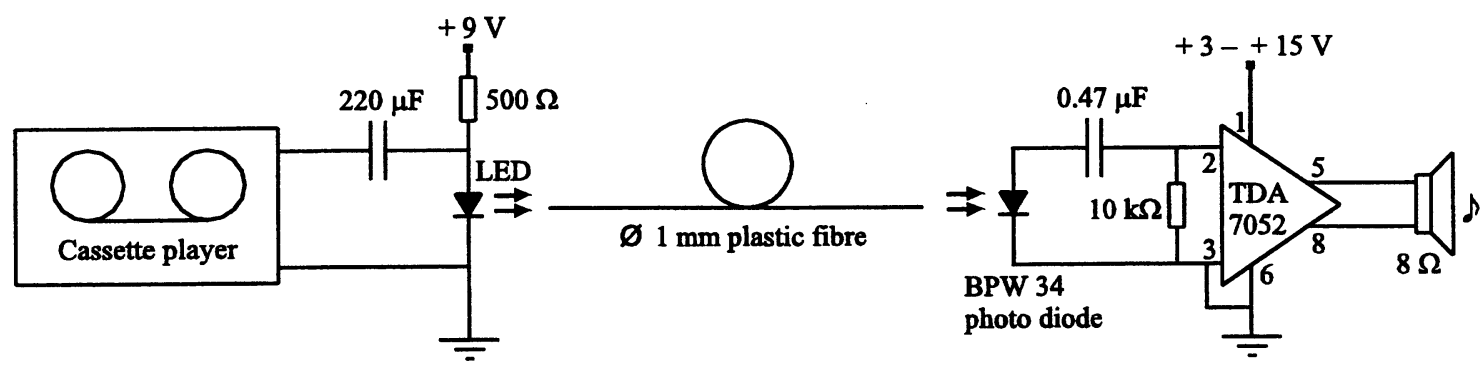

Fig. 1. Simple LED transmitter and receiver for demonstration of optical fiber transmission. Plastic optical fiber of $1 \mathrm{~mm}$ diameter is suitable.

In the transmitter, the signal source $\mathrm{AC}$ is superposed over the $\mathrm{DC}$ current from a battery and amplitude modulating the light from the LED. In the receiver, the photodiode works as a solar cell, the DC is blocked by the capacitor and the AC voltage over the resistor is amplified. 


\section{TOTAL REFLECTION}

To visualize total reflection in a step index fiber, a laser pointer and a Plexiglas bar with square cross section (25 $\times 25 \times 150 \mathrm{~mm}$ ) can be used. The end of the bar should be rounded to facilitate angle variations, see Fig. 2 . The incidence angle can then be varied up to the critical angle for total reflection. The back surface of the bar may be ground and the laser ray directed in a small angle towards that surface to increase the scattering of the light to the spectators. If the scattered area of the ray is moved forward by moving the laser slightly, it gives the impression of a pulse moving along the bar.

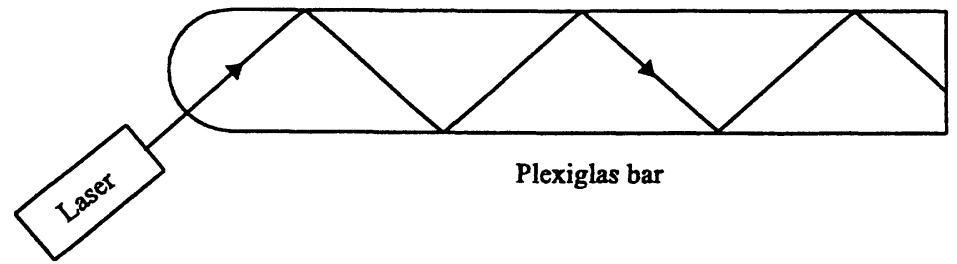

Fig. 2. A laser pointer and a Plexiglas bar can visualize total reflection, similar to that in a step index fiber core. The surrounding air works as low index cladding.

\subsection{Rayleigh scattering}

\section{LOSS MECHANISMS}

In a nearly dark room it can be seen how the straight fiber emits light due to Rayleigh scattering, the dominating loss mechanism in glass fibers, and important for understanding of reflectometry. It is an advantage to use a $635 \mathrm{~nm}$ laser as the eye is more sensitive to that wavelength than the usual longer wavelengths around $670 \mathrm{~nm}$ for laser pointers. One may emphasize that the fiber is visible due to the scattering loss, otherwise one cannot see light from the side.

\subsection{Bend loss}

A plastic fiber connected to a laser and bent to a small radius will emit light from the bend and give direct evidence of bend loss, which is important to know for cable installation personnel. The difference between a small circle $(\approx 10 \mathrm{~mm}$ in diameter) and a greater one $(\approx 40 \mathrm{~mm})$ is clearly visible. The direction of the light can be noticed in the tangential direction of the circles. The possibility to tap information from a bent optical fiber can be tested by the LED-link described above and a bent plastic fiber.

\subsection{Splice loss}

Two fibers, connected to the LED transmitter and receiver, can be placed with a small offset on the overhead projector table, where they can show splice loss as a function of transverse, longitudinal and angular offset.

\section{ACKNOWLEDGEMENT}

Many ideas and constructions were invented by Sven Ottosson, formerly with Ericsson Cables AB, now retired, and his contributions are gratefully acknowledged. 